\title{
Maternal autoantibodies and congenital heart block
}

\author{
Uma Pandey \\ Associate Professor, Dept. of Obstetrics and Gynaecology, Banaras Hindu University, Varanasi, Uttar Pradesh, India
}

Corresponding Author:

Email: uma.pandey2006@yahoo.com

\begin{abstract}
Introduction: Autoimmune congenital heart block $(\mathrm{CHB})$ of the fetus is the severest manifestation of neonatal lupus, and it is damage of AV node of the fetus that is the root cause problem. Congenital heart block is mainly associated with maternal Systemic Lupus Erythematous (SLE) and anti-Ro and anti-La positive maternal status. This observational study was done to know the incidence of congenital heart block in patients of Systemic Lupus Erythematous who had ANA, anti-Ro/SSA and anti$\mathrm{La} / \mathrm{SSB}$ levels positive.

Materials and Methods: A prospective observational study was done in a University teaching hospital of North India (Banaras Hindu University). The study duration was between January 2014 to December 2016 and 9 cases of SLE were identified and diagnosed. All these patients were tested for ANA, anti-Ro/SSA and anti- La/SSB antibodies, were found to be positive. Three fetuses had confirmed fetal heart rate abnormalities/congenital heart block. Our neonatologist treated fetuses with heart block as per their protocols. Mothers were counseled regarding recurrence of congenital heart block in the next pregnancy.

Results: All patients of SLE were positive for ANA, and had anti-Ro/ SSA- and anti-La/SSB-positive status. Three fetuses/neonate had congenital heart block, and one fetus required pacemaker after delivery.

Conclusions: Delivery should be organized in tertiary referral center and with multidisciplinary care. Treatment with pacemaker insertion is life saving. These fetuses can be salvaged if they are given proper care although couple should be explained the prognosis.
\end{abstract}

Keyword: Systemic Lupus Erythematous, Autoantibodies, Congenital heart block, Anti-Ro, anti-La, ANA, DSDNA, Lupus nephritis.

\section{Introduction}

The incidence of autoimmune disorder is approximately $4-5 \%$ in general population, being more prevalent in females. ${ }^{1}$

SLE is systemic connective tissue disease characterized by periods of disease activity and remissions. Ratio of female to male is 9:1 and during childbearing years it is $15: 1$. Average age of diagnosis is 30 years and $6 \%$ have other associated autoimmune disease. Joint involvement is commonest feature (90\%) although arthritis is non-erosive, peripheral and characterized by swelling and tenderness. Involvement of skin is there in $80 \%$ of cases in the form of malar rashes, discoidal rashes, photosensitivity, vasculitic lesions on fingertips, nail folds and discoidal lupus. These patients also suffer from Raynauds phenomenon.

Diagnosis is based on clinical and laboratory criteria of American Rheumatic Association. It includes blood examination showing normocytic, normochromic anemia, neutropenia and thrombocytopenia. In these cases ESR is raised because of high immunoglobulins levels. There is falling levels of C3 \& C4; and CRP remains normal.

ANA (Antinuclear antibodies) is the most common (96\%) antibody and titres don't change with disease activity over the time. Double stranded DNA (DSDNA) is very specific for SLE and positive in $80 \%$ of cases. Anti Ro, anti La are present in approximately $30 \%$ are associated with congenital heart block and fetal bradycardia, most lethal of the clinical manifestations.
Systemic Lupus Erythematous (SLE) starts at young age but it is the childbearing age women who are affected with greater magnitude due to its effect on pregnancy and reproductive outcomes. ${ }^{2}$

Pregnancy increases likelihood of flare from 40$60 \%$, may occur at any stage of pregnancy to puerperium. It most commonly involves skin and joints, more so if the disease is uncontrolled. If the disease is uncontrolled there is more incidence of miscarriage and fetal growth restriction. Flare may be difficult to diagnose during pregnancy because features like hair loss, oedema, palmar and facial erythema, fatigue, anaemia, musculoskeletal pain are also clinical symptoms and signs of normal pregnancy.

SLE has many adverse effects on pregnancy, it increase risk of miscarriage, intrauterine death, preeclampsia, prematurity and fetal growth restriction.

Pregnancy outcome is particularly affected by SLE induced renal disease; even early stage renal lupus is associated with increased intrauterine death, preeclampsia, prematurity and fetal growth restriction.

Congenital Heart Block (CHB) is due to damage to the atrioventricular nodes (AV node) by transplacental passage of antibodies. The incidence is $2-3 \%$ in cases of SLE. The recurrence is also high, almost 9 times. The onset of CHB is early on in second trimester. It is frequently associated with anti-Ro and anti-La antibodies. We did this study to know actual incidence of CHB in patients of SLE who had anti-Ro and anti-La antibodies. $^{3}$ 


\section{Materials and Methods}

This study was conducted prospectively and it is an observational study. It was conducted in a Sir Sunder Lal Hospital, University teaching hospital, Banaras Hindu University, of North India. Informed consent was taken from the pregnant women who were part of this study.

The study duration was from January 2014December 2016. The mothers had gestational scan at 810 weeks and anomaly scans/level 2 scans at 18-22 weeks of gestation. One fetus was found to have bradycardia at 28 weeks of gestation (it was observed in a peripheral hospital of Bihar state and documented in Ultrasound report). She was refereed from there and then investigated for autoimmune disorders/systemic lupus erythematous.

Total of 9 pregnant women were positive for ANA, DSDNA, anti Ro (SSA) and anti La (SSB) antibodies. A total of three fetuses were found to have congenital heat block. Fetal echocardiography was done in all three fetuses and structural cardiac anomaly was ruled out. Echocardiography was performed between 20-24 weeks gestation and then repeated once the bradycardia became evident. Fetal Doppler study performed on these fetuses showed normal parameter; there were no features of intrauterine fetal growth restriction.

Pregnant mothers were asked to continue her previous SLE medications in consultation with physicians, and they were continued throughout pregnancy. Gestational age was confirmed by dates and correlated with ultrasonography. Maternal and neonatal data was collected on a proforma.

\section{Results}

All patients (9) of SLE were positive for ANA, DSDNA, and had anti-Ro/ SSA- and anti-La/SSBpositive status. Three fetuses/neonate had congenital heart block, and one fetus required pacemaker after delivery in the fifth month. The age range of mothers was between $25-37$ years, $44 \%$ of mothers were primigravida and they were of middle socioeconomic status.

Congenital heart block was diagnosed by echocardiography and heart rate tracing in the Ultrasound and Cardiotocogram in two fetuses at 24 and 29 weeks period of gestation, respectively. One fetus was found to have bradycardia from 28 weeks of gestation (it was observed in a peripheral hospital of Bihar state and documented in Ultrasound report). 65.55\% mothers were delivered by Caesarean section and rests were normal delivery.

Heart rate of these fetuses ranged between 52-76/min. Birth weight was $>2.5 \mathrm{~kg}$ in two fetuses but the only fetus that had bradycardia at 28 weeks of gestation in Bihar had weight of $<2 \mathrm{~kg}$ only, as the mother also had Rheumatic heart diseases with mitral stenosis and pulmonary hypertension. Apgar score of this neonate was 5 at 1 minute and 7 at 5 minutes. This baby required ventilatory support and recovered well. Pacemeker insertion was done in the fifth month of life.

\section{Discussion}

SLE is more common among women than men worldwide. Healthcare sector is not as well developed in low resource setting as it is in rich nations. Due to this reason majority of our women were diagnosed to have SLE during pregnancy, although the incidence of SLE in pregnancy worldwide is 1 in $1250 .^{4}$

It is important to counsel the pregnant women or those who are planning for pregnancy that SLE without hypertension and renal involvement has almost similar outcome to that of general population. Anti Ro and Anti La positive mothers have risk of transient neonatal cutaneous lupus 5\% and congenital heart block almost $2 \%$.

During pregnancy, the maternal antibodies cross the placenta and bind to the atrioventricular (AV) conduction system with subsequent fibrosis and calcification leading to a complete AV block. ${ }^{5}$ Neonatal Lupus Syndrome is due to autoantibodies crossing the placenta that are directed against cytoplasmic ribonucleoproteins. Cutaneous form of neonatal lupus is milder one but congenital heart block could be lethal. Risk of neonatal lupus is increased to $20 \%$ if previous one child is affected; it increases $50 \%$ if previous 2 children are affected.

Fetal circulation is established by 12 weeks of gestation but congenital heart block is not diagnosed until 16-32 weeks gestation. Most common presenting symptom is fetal bradycardia diagnosed on ultrasound examination or fetal heart rate auscultation/cardiotocogram. Detailed cardiac scanning shows atrio-ventricular dissociation, which is diagnostic of congenital heart block. Unless managed properly mortality rates in these fetuses are very high. It is caused by inflammation and fibrosis of the conducting system. Maternal antibodies initiate trans differentiation of cardiac fibroblasts to unchecked proliferating myofibroblasts, this causes scarring of atrioventricular node. It usually happens that heart block progresses from first and second degree heart block to third degree heart block.

All patients (9) of SLE were positive for ANA, DSDNA, and had anti-Ro/ SSA- and anti-La/SSBpositive status. Three fetuses/neonate had congenital heart block, and one fetus required pacemaker after delivery in the fifth month. During pregnancy, the antibodies present in the maternal circulation can cross the placenta and affect the fetus.

Maternal administration of intravenous immunoglobulin and corticosteroids are not effective in cases of Congenital heart block. PITCH study showed that IVIG at $400 \mathrm{mg} / \mathrm{kg}$ on a tri-weekly schedule from 12 to 24 weeks of gestation is ineffective as a prevention of Congenital heart block in pregnancies at risk of recurrence. Regular and close monitoring for 
heart rate and pacemaker insertion in heart is beneficial which was done in this study. ${ }^{6}$

Temporary pacing can be achieved transcutaneously, transesophageally or transvenously in the neonate. A permanent pacemaker placement may be needed in many of the neonates of $\mathrm{CHB}$ depending upon their cardiac performance. It is prudent to refer the neonate to the facility where pacemaker insertion can be done. Involvement of Cardiologist who deals with neonates is very helpful. In our study, the fetus that had bradycardia at 28 weeks of gestation, pacemaker was needed. ${ }^{7}$

\section{Conclusion}

Treatment with dexamethasone has been tried but it's not very effective. It may reverse the lesser degree of heart block partially but progression to complete heart block is usually seen. Complete heart block needs pacemaker. $19 \%$ of the affected babies expire in the early neonatal period and almost $50-60 \%$ of the infants require pacemaker.

Women with lupus nephritis or Anti-phospholipid antibody should be treated with low dose aspirin during pregnancy. Fetal growth monitoring by uterine artery Doppler at 20-24 weeks gestation and umbilical artery Doppler from 24 weeks gestation should be done.

In modern times the prognosis of fetuses/neonates of mothers with SLE have improved with intensive fetal monitoring, echocardiography and pacemakers insertion. Steroids and immunoglobulins have have its side effects on the mother and we did not try in our study. Parents should be extensively counseled and told the importance of follow up. Prognosis of baby is not grim but chances of recurrence if very high.

Compliance with Ethical Standards

Conflict of interest: no conflict of interest.

Informed Consent: was obtained from all patients for whom data was collected on the proforma

\section{References}

1. Wandstrat A, Wakeland E. The genetics of complex autoimmune diseases: non-MHC susceptibility genes. Nat Immunol. 2001;2:802-9.

2. Petri M. Epidemiology of systemic lupus erythematosus. Best Pract Res Clin Rheumatol. 2002;16:847-8.

3. Carolis SD, Salvi S, Botta A, et al. which intrauterine treatment for autoimmune congenital heart block? Open Autoimmune J. 2010;2:1-10.

4. Clowes ME, Jamison M, Myers E, et al. A national study of the complications of lupus in pregnancy. Am J Obstet Gynecol. 2008;199:127e1.

5. Nield LE, Silverman ED, Smallhorn JF, et al. Endocardial fibroelastosis associated with maternal anti-Ro and antiLa anti- bodies in the absence of atrioventricular block. Circulation. 2002;40:796-802.

6. Friedman DM, Llanos C, Izmirly PM, et al. Evaluation of fetuses in the preventive IVIG therapy for congenital heart block (PITCH) study. Arthritis Rheum. 2010;62(4):1138-46.

7. Puria S, Pooni P, Mohan B, et al. Pregnancy with SLE and fetal congenital heart block: a case report. Cardiol Res. 2013;4:126-8. 